IC/2000/XX

hep-th/0008132

\title{
Noncommutative QED and Anomalous Dipole Moments
}

\author{
Ihab. F. Riad and M.M. Sheikh-Jabbari \\ The Abdus Salam International Centre for Theoretical Physics \\ Strada Costiera, 11. 34014, Trieste, Italy \\ ifriad, jabbari@ictp.trieste.it
}

\begin{abstract}
We study QED on noncommutative spaces, NCQED. In particular we present the detailed calculation for the noncommutative electron-photon vertex and show that the Ward identity is satisfied. We discuss that in the noncommutative case moving electron will show electric dipole effects. In addition, we work out the electric and magnetic dipole moments up to one loop level. For the magnetic moment we show that noncommutative electron has an intrinsic (spin independent) magnetic moment.
\end{abstract}

\footnotetext{
${ }^{1}$ A Dissertation Presented by I.F.R. to the ICTP High Energy Section in Candidacy for the Diploma Degree, August 2000
} 


\section{Contents}

$\begin{array}{lll}1 & \text { Introduction } & 2\end{array}$

\begin{tabular}{|lll}
\hline 2 & Preliminaries & 3
\end{tabular}

2.1 Noncommutative spaces . . . . . . . . . . . . . . . . . . . . . . 4

2.2 The Noncommutative QED . . . . . . . . . . . . . . . . . . . . . . . . . 5

2.3 Feynman rules for NCQED . . . . . . . . . . . . . . . . . . . . . . . 7

\begin{tabular}{|lll}
3 & Electron-photon vertex at one loop level & 9
\end{tabular}

3.1 Vertex structure at the one loop level . . . . . . . . . . . . . . . . . . . . . . 9

3.2 Renormalization . . . . . . . . . . . . . . . . . . . 14

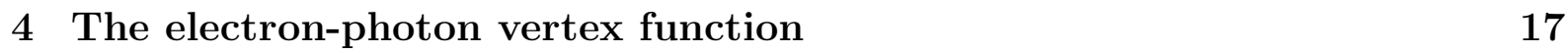

$4.1 \quad$ Electron-photon vertex in usual QED . . . . . . . . . . . . . . . . . . . . 17

4.2 Dipole moment at the tree level in NCQED . . . . . . . . . . . . . . . . . 20

4.3 The electron-photon vertex structure in NCQED . . . . . . . . . . . . . . . 20

\begin{tabular}{|lr}
5 Concluding remarks & 23
\end{tabular}

\begin{tabular}{|ll}
\hline A Some useful identities in $*^{*}$-product calculus & 24
\end{tabular}

\section{Introduction}

Although, physics on the noncommutative spaces has a long standing story [1] , recently it has been re-motivated by string theory arguments [2]. Apart from the string theory interests the field theories on such spaces, noncommutative field theories (NCFT's), in their own turn are very interesting. Although being non-local, it has been argued that they can be treated as sensible field theories, and in the last two years there have been a lot of work devoted to the study of NCFT's. The question of renormalizability of NCFT's in general and in particular NC scalar and NC Yang-Mills (NCYM) theories have been 


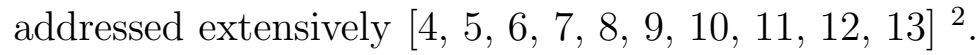

For the NC scalar theories it has been shown that real $\Phi^{4}$ theory in 4 dimensions is two loop renormalizable [5, 13]. The pure NCYM have been studied only in one loop level and shown to be renormalizable. The $\beta$-function of these theories (pure NCYM) is found to be the same as the corresponding commutative theory.

The problem of adding fermions (matter fields) has not been studied in detail. In particular, noncommutative version of QED, NCQED, has been discussed in [8, 10, 11, 14, 15]. We emphasize that here we only consider spaces-like noncommutativities and not the time-like one (noncommutative space-times). In the latter case, it has been shown that the corresponding field theories are not unitary [16]. However, for the light-like noncommutativity it has been shown that we still have a well-defined quantum theory [17.

In this work we study some details of NCQED. Working out the electron-photon interaction vertex in NCQED up to one loop, we find the so-called vertex functions and thereby we read off the anomalous magnetic moment. By explicit calculation we show that Ward identity is satisfied in the NCQED case. Since we have an extra suitable vector in our theory, there is some room for new type of magnetic moment which is spin independent and is proportional to the noncommutativity parameter.

As it has been discussed in [11, 18] particles in the noncommutative gauge theories show electric dipole effects. We recall this property in the classical case, and also study one loop quantum corrections to the electric dipole moment.

The paper is organized as following. In section 2, we briefly review the preliminaries we need; we introduce noncommutative spaces and field theories in general and specify the NCQED by presenting its classical action and basic Feynman graphs. Then in section 3, we work out details of noncommutative electron-photon vertex at one loop level. In addition, renormalizing the corresponding graphs in the minimal subtraction (MS) scheme, we discuss the IR/UV mixing which is a characteristic of any NCFT. In section 4 , using the renormalized vertex functions we find "electromagnetic dipole" properties of noncommutative electron. We end this work by conclusions and remarks.

\section{Preliminaries}

\footnotetext{
${ }^{2}$ Because of the huge number of papers on this issue, hereby we apologize for all the related works which have not been quoted.
} 


\subsection{Noncommutative spaces}

Usual quantum mechanics is formulated on commutative spaces satisfying the following commutation relations,

$$
\begin{array}{rrr}
{\left[\hat{X}_{i}, \hat{P}_{j}\right]=} & i \hbar \delta_{i j} \quad \text { and } \\
{\left[\hat{X}_{i}, \hat{X}_{j}\right]=0 \quad,} & {\left[\hat{P}_{i}, \hat{P}_{j}\right]=0 .}
\end{array}
$$

Then in order to describe a noncommutative space, the above commutation relations should be changed as,

$$
\begin{aligned}
{\left[\hat{X}_{i}, \hat{P}_{j}\right] } & =i \hbar \delta_{i j}, \\
{\left[\hat{X}_{i}, \hat{X}_{j}\right] } & =i \theta_{i j} \quad \text { and } \\
{\left[\hat{P}_{i}, \hat{P}_{j}\right] } & =0
\end{aligned}
$$

where $\theta_{i j}$, is the noncommutative constant of dimension $[M]^{-2}$. We can see that for theories on such spaces the Lorentz symmetry is explicitly violated however, it will be recovered in the $\theta \rightarrow 0$ limit. The above noncommutative theory can be expanded to include noncommuting space-time, i.e.

$$
\left[\hat{X}_{\mu}, \hat{X}_{\nu}\right]=i \theta_{\mu \nu}
$$

These type of theories were studied in [16] and shown to suffer from the loss of unitarity, so for the purpose of our work here, we will only consider the noncommuting spaces. Since our field theory is better formulated through the path integral formulation, we can implement the noncommutivity of space into path integral formulation through what is known as the Weyl-Moyal correspondence [12]

$$
\begin{gathered}
\hat{\Phi}(\hat{X}) \longleftrightarrow \Phi(x) ; \\
\hat{\Phi}(\hat{X})=\int_{\alpha} e^{i \alpha \hat{X}} \phi(\alpha) d \alpha, \\
\phi(\alpha)=\int e^{-i \alpha x} \Phi(x) d x,
\end{gathered}
$$

\footnotetext{
${ }^{3}$ In the following equations there are some obvious factors of $2 \pi$ which we avoid them here.
} 
where $\alpha$ and $x$ are real variables. Then,

$$
\begin{aligned}
\hat{\Phi}_{1}(\hat{X}) \hat{\Phi}_{2}(\hat{X}) & =\iint_{\alpha \beta} e^{i \alpha \hat{X}} \phi(\alpha) e^{i \beta \hat{X}} \phi(\beta) d \alpha d \beta \\
& =\iint_{\alpha \beta} e^{i(\alpha+\beta) \hat{X}-\frac{1}{2} \alpha_{\mu} \beta_{\nu}\left[\hat{X}_{\mu}, \hat{X}_{\nu}\right]} \phi(\alpha) \phi(\beta) d \alpha d \beta
\end{aligned}
$$

hence

$$
\begin{gathered}
\hat{\Phi}_{1}(\hat{X}) \hat{\Phi}_{2}(\hat{X}) \longleftrightarrow\left(\Phi_{1} * \Phi_{2}\right)(x), \\
\left(\Phi_{1} * \Phi_{2}\right)(x) \equiv\left[e^{\frac{i}{2} \theta_{\mu \nu} \partial_{\zeta \mu} \partial_{\eta \nu}} \Phi(x+\zeta) \Phi(x+\eta)\right]_{\zeta=\eta=0} .
\end{gathered}
$$

In other words, the noncommutative version of a field theory is obtained by replacing all the field products by the star product (2.7). It is easy to check that the Moyal bracket of coordinates defined by,

$$
\left[x_{\mu}, x_{\nu}\right]_{M B}=x_{\mu} * x_{\nu}-x_{\nu} * x_{\mu}
$$

satisfies the commutation relations on the noncommutative spaces (2.3).

\subsection{The Noncommutative QED}

In this part we introduce the structure of the action for NCQED [8]. We will notice that due to the presence of the star product and the Moyal brackets, the noncommutative U(1) is similar to non-Abelian gauge theories. As usual, the action for a gauge theory consists of two parts, the gauge fields and matter fields, fermions. To start with, we write the term for the gauge fields:

$$
S_{Y M}=-\int d^{4} x \frac{1}{4 e^{2}} F_{\mu \nu} * F^{\mu \nu}
$$

with

$$
F_{\mu \nu}=\partial_{\mu} A_{\nu}-\partial_{\nu} A_{\mu}-i\left[A_{\mu}, A_{\nu}\right]_{M B}
$$

The above action enjoys the noncommutative $\mathrm{U}(1)$ symmetry, defined by

$$
\begin{aligned}
A_{\mu} \rightarrow A_{\mu}^{\prime}(x) & =U(x) * A_{\mu} * U(x)^{-1}+i U(x) * \partial_{\mu} U(x)^{-1} \\
U(x) & =\exp *(i \lambda) \equiv 1+i \lambda-\frac{1}{2} \lambda * \lambda+\ldots .
\end{aligned}
$$


Under the above transformation, the field strength $F_{\mu \nu}$, transforms as

$$
F_{\mu \nu} \rightarrow F_{\mu \nu}^{\prime}=U(x) * F_{\mu \nu} * U(x)^{-1}
$$

and hence

$$
S_{Y M} \rightarrow S_{Y M}^{\prime}=-\int d^{4} x \frac{1}{4 e^{2}} U(x) * F_{\mu \nu} * U(x)^{-1} * U(x) * F^{\mu \nu} * U(x)^{-1} .
$$

using,

$$
U(x) * U(x)^{-1}=U(x)^{-1} * U(x)=1,
$$

and the cyclic property of the star product under the integral (see appendix A), we conclude that (2.9) is invariant under the above defined gauge transformation. Also using the property that under the integral the star product is commuting, one can remove the star product between two $F$ 's, then

$$
S_{Y M}=-\int d^{4} x \frac{1}{4 e^{2}} F_{\mu \nu} F^{\mu \nu} .
$$

In order to add the matter fields, we need the notion of covariant derivative, $D_{\mu}$, for which we demand that our fermionic sector is also invariant under the above mentioned noncommutative local gauge transformations. We notice that one can extend the local gauge transformations to fermions in the following two different ways,

$$
\Psi(x) \rightarrow \Psi^{\prime}(x)=U(x) * \Psi(x)
$$

or

$$
\tilde{\Psi}(x) \rightarrow \tilde{\Psi}^{\prime}(x)=\tilde{\Psi} * U(x)^{-1}
$$

Therefore, one can define two covariant derivatives corresponding to two different fermions:

$$
D_{\mu} \Psi=\partial_{\mu} \Psi-i A_{\mu} * \Psi
$$

or

$$
D_{\mu} \tilde{\Psi}=\partial_{\mu} \tilde{\Psi}+i \tilde{\Psi} * A_{\mu}
$$

From the above two equations we see that by taking the limit, $\theta_{\mu \nu} \rightarrow 0$, the star product disappears and the two fermion fields $\Psi(x)$ and $\tilde{\Psi}(x)$ will show different charges. In fact 
it has been shown that these two fermions are related by the noncommutative version of the charge conjugation [14].

So, altogether the full Lagrangian can be written as,

$$
S=\int d^{4} x\left(-\frac{1}{4 e^{2}} F_{\mu \nu} F^{\mu \nu}+\bar{\Psi}(\not D-m) \Psi+L_{\text {gauge }}+L_{\text {ghost }}\right),
$$

where we have also added the term $L_{\text {gauge }}$ for gauge fixing. Since the quadratic terms are the same as in the usual QED, we have the same gauge fixing term. The $L_{\text {ghost }}$ accounts for the ghost fields appearing in the gauge fixing procedure, similar to that of the non-Abelian gauge theories.

\subsection{Feynman rules for NCQED}

Using the property of the star product under the integral sign and that, the quadratic terms are not changed in the presence of the star product, we conclude that the propagators for the free fermion, gauge and the ghost fields are the same as in the case of usual QED, i.e.

$$
\begin{aligned}
\overbrace{\mathrm{P}} & =\frac{i}{\not p-m+i \epsilon}, \\
& =\frac{g^{\mu \nu}}{i\left(p^{2}+i \epsilon\right)}, \\
& =\frac{-1}{i\left(p^{2}+i \epsilon\right)},
\end{aligned}
$$

where we were using the Feynman gauge.

For the interaction terms, we see that they are similar to those of non-Abelian gauge theories [4, 8], in the sense that we get cubic and quadric interaction vertices for the gauge

fields besides the usual vertices found usual QED. Here we just present the Feynman rules, to show their similarities and differences with the non-Abelian case. 

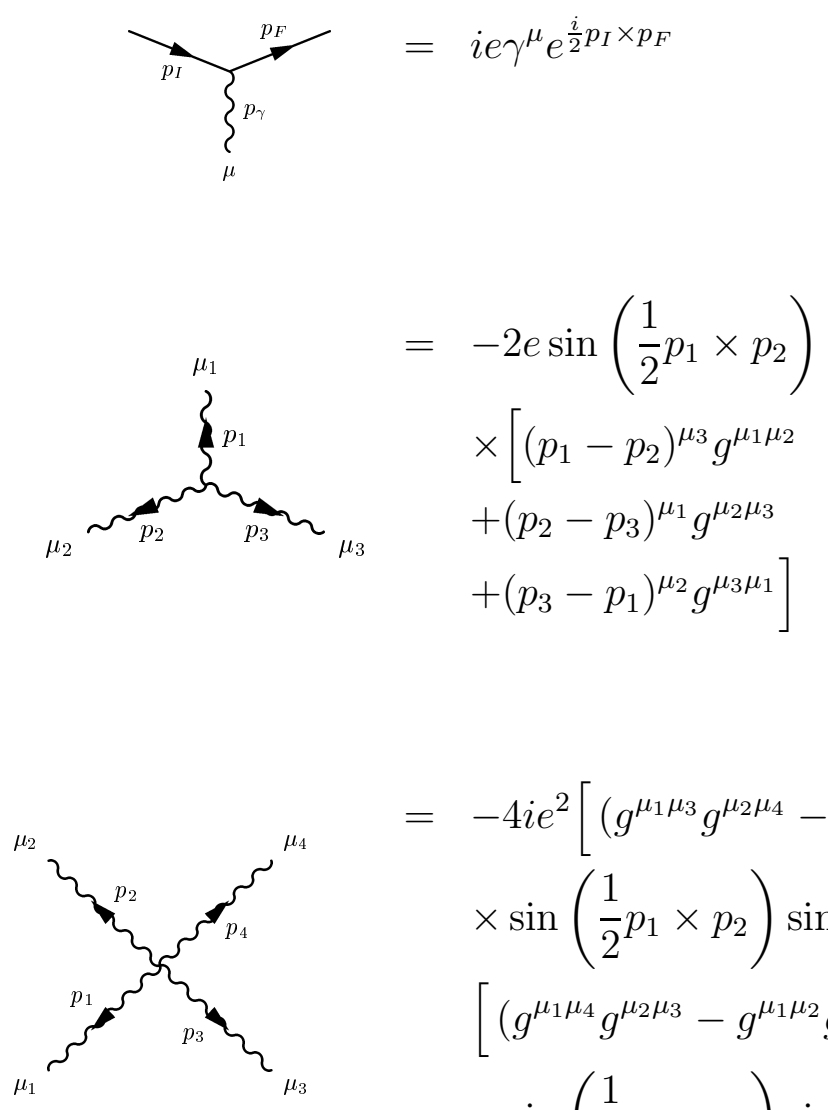

$$
\begin{aligned}
= & -4 i e^{2}\left[\left(g^{\mu_{1} \mu_{3}} g^{\mu_{2} \mu_{4}}-g^{\mu_{1} \mu_{4}} g^{\mu_{2} \mu_{3}}\right)\right. \\
& \times \sin \left(\frac{1}{2} p_{1} \times p_{2}\right) \sin \left(\frac{1}{2} p_{3} \times p_{4}\right) \\
& {\left[\left(g^{\mu_{1} \mu_{4}} g^{\mu_{2} \mu_{3}}-g^{\mu_{1} \mu_{2}} g^{\mu_{3} \mu_{4}}\right)\right.} \\
& \times \sin \left(\frac{1}{2} p_{3} \times p_{1}\right) \sin \left(\frac{1}{2} p_{2} \times p_{4}\right) \\
& {\left[\left(g^{\mu_{1} \mu_{2}} g^{\mu_{3} \mu_{4}}-g^{\mu_{1} \mu_{3}} g^{\mu_{2} \mu_{4}}\right)\right.} \\
& \times \sin \left(\frac{1}{2} p_{1} \times p_{4}\right) \sin \left(\frac{1}{2} p_{2} \times p_{3}\right)
\end{aligned}
$$

$$
\overrightarrow{p_{I}}-\underbrace{-\overrightarrow{p_{F}}}_{\mu}-\operatorname{siep}_{F}^{\mu} \sin \left(\frac{1}{2} p_{I} \times p_{F}\right) .
$$

We observe that all vertices here are similar to those in non-Abelian gauge theories in which the structure constant is replaced by $2 \sin \left(\frac{1}{2} p \times p^{\prime}\right)$. This can be seen if we notice that the structure constants appear because of the commutation $\left[A_{a}, A_{b}\right]=i f_{a b c} A^{c}$ in non-Abelian theories. Hence we expect the appearance of the factor $2 \sin \left(\frac{1}{2} p \times p^{\prime}\right)$ as a consequence of the Moyal bracket, i.e. 


$$
\begin{aligned}
{\left[A_{\mu}, A_{\nu}\right]_{M B} } & =A_{\mu} * A_{\nu}-A_{\nu} * A_{\mu} \\
& =\int d^{4} p d^{4} p^{\prime} A_{\mu}(p) A_{\nu}\left(p^{\prime}\right)\left(e^{\frac{i}{2} p \times p^{\prime}}-e^{\frac{-i}{2} p \times p^{\prime}}\right) e^{i\left(p+p^{\prime}\right) \cdot x} \\
& =\int d^{4} p d^{4} p^{\prime} 2 i A_{\mu}(p) A_{\nu}\left(p^{\prime}\right) \sin \left(\frac{1}{2} p \times p^{\prime}\right) e^{i\left(p+p^{\prime}\right) \cdot x} .
\end{aligned}
$$

\section{Electron-photon vertex at one loop level}

In the previous section we introduced the NCQED, and showed that in this theory there are new type of vertices similar to those found in non-Abelian gauge theories. In this section we perform explicit calculation of the vertex function for the photon-electron at the one loop level which are expected to contribute to the anomalous magnetic moment.

\subsection{Vertex structure at the one loop level}

In the case of NCQED and because of the three photon vertices, the electron-photon vertex receives contributions from the two diagrams of figure.1 .

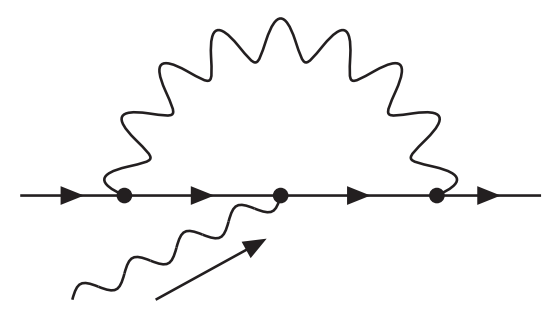

(a) One loop QED-like correction to $\psi \bar{\psi} A_{\mu}$

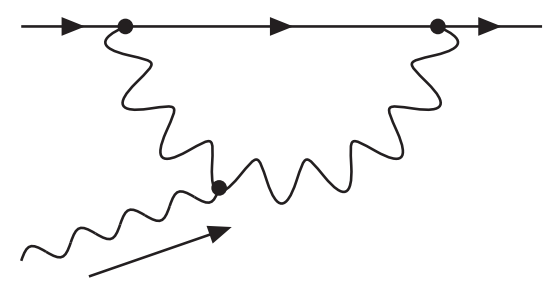

(b) Nonabelian-type correction to $\psi \bar{\psi} A_{\mu}$

Figure 1: One loop correction to $\psi \bar{\psi} A_{\mu}$ vertex 
The first diagram is similar to, what can also be found in the usual QED however, the second diagram is completely new. Now we proceed to write the analaytic expression of the first diagram,

$$
\begin{aligned}
\Gamma_{\mu}^{(a)}= & i(-i e)^{2} e^{\frac{i}{2} p \times p^{\prime}} \int \frac{d^{4} k}{(2 \pi)^{4}} e^{-i k \times q} \frac{\gamma_{\sigma}}{k^{2}-m_{\gamma}^{2}+i \epsilon} \frac{\not p^{\prime}-\not k+m}{\left(p^{\prime}-k\right)^{2}-m^{2}+i \epsilon} \gamma_{\mu} \\
& \frac{\not p-\not k+m}{(p-k)^{2}-m^{2}+i \epsilon} \gamma^{\sigma},
\end{aligned}
$$

where $m_{\gamma}$ is a photon mass accounting for the IR divergences.

Using the on mass shell conditions the numerator in the above expression may be written as

$$
4\left\{\gamma_{\mu}\left[\left(p^{\prime}-k\right)(p-k)-\frac{k^{2}}{2}\right]+\not k\left(p^{\prime}+p-k\right)_{\mu}-m k_{\mu}\right\}
$$

To compute the above integral, we replace the propagators by the Schwinger parameters:

$$
\frac{i}{p^{2}-m^{2}+i \epsilon}=\int_{0}^{\infty} d \alpha e^{i \alpha\left(p^{2}-m^{2}+i \epsilon\right)}
$$

and use the auxiliary integral,

$$
\begin{gathered}
\int \frac{d^{4} k}{(2 \pi)^{4}} e^{i k .(z-\tilde{q})} \frac{1}{\left(k^{2}-m_{\gamma}^{2}\right)\left(k^{2}-2 p^{\prime} . k\right)\left(k^{2}-2 p . k\right)}= \\
=\frac{1}{(4 \pi)^{2}} \int_{0}^{\infty} d \alpha_{1} d \alpha_{2} d \alpha_{3} \frac{\exp \left[\frac{-i}{a}\left(\left(\alpha_{1}+\alpha_{2}+\alpha_{3}\right) \alpha_{1} m_{\gamma}^{2}+\left(\frac{(z-\tilde{q})}{2}-\alpha_{2} p^{\prime}-\alpha_{3} p\right)^{2}\right)\right]}{\left(\alpha_{1}+\alpha_{2}+\alpha_{3}\right)^{2}}
\end{gathered}
$$

where

$$
\tilde{q}_{\nu}=\theta_{\mu \nu} q^{\mu}
$$

and $\quad a=\alpha_{1}+\alpha_{2}+\alpha_{3}$. The introduction of the factor $e^{i k . z}$ in the integrand allows us to obtain the required expression in the numerator of $\Gamma_{\mu}$ by differentiation [21]. After 
symmetrization in $\alpha_{2}$ and $\alpha_{3}$ we obtain,

$$
\begin{aligned}
\Gamma_{\mu}^{(a)=} & \frac{\alpha}{i \pi} e^{\frac{i}{2} p \times p^{\prime}} \int_{0}^{\infty} d \alpha_{1} d \alpha_{2} d \alpha_{3} \frac{e^{\frac{i}{a \Lambda_{e f f}^{2}}}}{a^{3}} \exp \left[\frac{-i}{a}\left(\alpha_{1} a m_{\gamma}^{2}+\left(\alpha_{2}+\alpha_{3}\right)^{2} m^{2}-\alpha_{2} \alpha_{3} q^{2}\right)\right] \\
& e^{\frac{-i\left(\alpha_{2}+\alpha_{3}\right) p \cdot \tilde{q}}{a}}\left\{\gamma_{\mu}\left[a p^{\prime} \cdot p-\frac{\left(\alpha_{2}+\alpha_{3}\right)\left(p+p^{\prime}\right)^{2}}{2}+\frac{i}{2}+\frac{\left(m^{2}\left(\alpha_{2}+\alpha_{3}\right)^{2}-\alpha_{2} \alpha_{3} q^{2}\right)}{2 a}\right]\right. \\
+ & \frac{m}{2 a}\left(p^{\prime}+p\right)_{\mu} \alpha_{1}\left(\alpha_{2}+\alpha_{3}\right)-\frac{\gamma_{\mu}}{4 a}\left(\left(p^{\prime}+p\right)\left(\alpha_{1}+\alpha_{1}+\alpha_{2}+\alpha_{3}\right)-\frac{1}{2} \tilde{q}\right) \cdot \tilde{q}+ \\
& \left.+\frac{2 m\left(\alpha_{1}+2 \alpha_{2}+2 \alpha_{3}\right) \tilde{q}_{\mu}+\left(\left(p^{\prime}+p\right)\left(2 \alpha_{1}+\alpha_{2}+\alpha_{3}\right)-\tilde{q}\right)_{\mu} \gamma \cdot \tilde{q}}{4 a}\right\}
\end{aligned}
$$

where we have inserted a UV regulator, $\exp \left(\frac{i}{a \Lambda^{2}}\right)$, with the following notation ff,

$$
\Lambda_{e f f}^{2}=\frac{1}{\Lambda^{-2}-\frac{\tilde{q} \cdot \tilde{q}}{4}}
$$

After the Wick rotation, i.e $\alpha_{i} \rightarrow \frac{\alpha_{i}}{i}$, with the help of the following identity,

$$
1=\int_{0}^{\infty} d \rho \delta\left(\rho-\sum \alpha_{i}\right)
$$

and rescaling $\alpha_{i} \rightarrow \rho \alpha_{i},(3.6)$ is obtained to be

$$
\begin{aligned}
\Gamma_{\mu}^{(a)}= & \frac{-\alpha}{\pi} e^{\frac{i}{2} p \times p^{\prime}} \int_{0}^{1} d \alpha_{1} d \alpha_{2} d \alpha_{3} \delta\left(1-\sum \alpha_{i}\right) e^{-i\left(\alpha_{2}+\alpha_{3}\right) p \cdot \tilde{q}} \times\left(A_{\mu}+\frac{B_{\mu}}{\rho}+\frac{C_{\mu}}{\rho^{2}}\right) \\
& \times \int_{0}^{\infty} d \rho \exp \left(-\rho\left(\alpha_{1} m_{\gamma}^{2}+\left(\alpha_{2}+\alpha_{3}\right)^{2} m^{2}-\alpha_{2} \alpha_{3} q^{2}\right)-\frac{1}{\rho \Lambda_{\text {eff }}^{2}}\right)
\end{aligned}
$$

where,

$$
\begin{gathered}
A_{\mu}=\gamma_{\mu}\left[p^{\prime} \cdot p-\frac{\left.\left(\alpha_{2}+\alpha_{3}\right)\left(p^{\prime}+p\right)^{2}\right)}{2}+\frac{1}{2} m^{2}\left(\alpha_{2}+\alpha_{3}\right)^{2}-\frac{1}{2} \alpha_{2} \alpha_{3} q^{2}\right]+ \\
\frac{m}{2} \alpha_{1}\left(\alpha_{2}+\alpha_{3}\right)\left(p^{\prime}+p\right)_{\mu}, \\
i B_{\mu}=\frac{\gamma_{\mu}}{2 i}+\frac{\gamma_{\mu} p \cdot \tilde{q}}{2}\left(2-\alpha_{2}-\alpha_{3}\right)+\frac{m}{2}\left(1+\alpha_{2}+\alpha_{3}\right) \tilde{q}_{\mu}+\frac{1}{4}\left(\alpha_{1}+1\right)\left(p^{\prime}+p\right)_{\mu} \gamma \cdot \tilde{q}, \\
C_{\mu}=\frac{-\gamma_{\mu} \tilde{q} \cdot \tilde{q}}{8}+\frac{\tilde{q}_{\mu} \gamma \cdot \tilde{q}}{4} .
\end{gathered}
$$

\footnotetext{
${ }^{4}$ We should note that due to anti-symmetry of $\theta_{\mu \nu}, \tilde{q} \cdot \tilde{q}$ is negative valued.
} 
Performing the integral over $\rho$, we get

$$
\begin{aligned}
\Gamma_{\mu}^{(a)}= & \frac{-\alpha}{\pi} e^{\frac{i}{2} p \times p^{\prime}} \int_{0}^{1} d \alpha_{1} d \alpha_{2} d \alpha_{3} \delta\left(1-\sum \alpha_{i}\right) e^{-i\left(\alpha_{2}+\alpha_{3}\right) p . \tilde{q}} \\
& \left(\frac{2 A_{\mu} K_{1}(2 \sqrt{X})}{\sqrt{X} \Lambda_{e f f}^{2}}+2 B_{\mu} K_{0}(2 \sqrt{X})+2 \sqrt{X} \Lambda_{\text {eff }}^{2} C_{\mu} K_{1}(2 \sqrt{X})\right),
\end{aligned}
$$

where

$$
X \equiv \frac{\alpha_{1} m_{\gamma}^{2}+\left(\alpha_{2}+\alpha_{3}\right)^{2} m^{2}-\alpha_{2} \alpha_{3} q^{2}}{\Lambda_{e f f}^{2}},
$$

and $K_{0}, K_{1}$ are the modified Bessel functions of the first and second type respectively.

Now we consider the contribution from the second diagram, the analytic expression of which reads as

$$
\begin{gathered}
\Gamma_{b}^{\mu}=-i e^{2} e^{\frac{i}{2} p \times p^{\prime}} \int \frac{d^{4} k}{(2 \pi)^{4}} \frac{\left(1-e^{i k \cdot \tilde{q}} e^{i p^{\prime} \times p}\right)}{\left(k^{2}-m^{2}\right)\left(\left(p^{\prime}-k\right)^{2}-m_{\gamma}^{2}\right)\left((p-k)^{2}-m_{\gamma}^{2}\right)} \times \\
\times\left\{\gamma_{\nu}(\not k+m) \gamma_{\rho}\left[g^{\mu \nu}\left(2 p^{\prime}-p-k\right)^{\rho}+g^{\nu \rho}\left(2 k-p^{\prime}-p\right)^{\mu}+g^{\rho \mu}\left(2 p-p^{\prime}-k\right)^{\nu}\right]\right\} .
\end{gathered}
$$

Using the gamma matrices algebra and the on shell condition the numerator can be written as,

$$
8 m k_{\mu}-2 \not k\left(p^{\prime}+p+2 k\right)_{\mu}+2 \gamma_{\mu}\left(2 p^{\prime} . k+2 p . k-k^{2}-3 m^{2}\right) .
$$

At this point we notice that (3.15) can be separated into two parts, one containing the phase $e^{-i k . \tilde{q}}$, and the other not, i.e. $\Gamma_{\mu}^{b}=\Gamma_{\mu}^{b 1}+\Gamma_{\mu}^{b 2}$. First we perform the part containing the phase, $\Gamma_{\mu}^{(b 2)}$, then the other term, $\Gamma_{\mu}^{(b 1)}$, can be recovered easily.

Following the same spirit of the previous calculation, the auxiliary integral is introduced and then doing the Gaussian integral,

$$
\begin{aligned}
& \int \frac{d^{4} k}{(2 \pi)^{4}} \frac{e^{i k \cdot(z+\tilde{q})}}{\left(k^{2}-m^{2}\right)\left(\left(p^{\prime}-k\right)^{2}-m_{\gamma}^{2}\right)\left((p-k)^{2}-m_{\gamma}^{2}\right)}= \\
& \frac{1}{4 \pi^{2}} \int_{0}^{\infty} d \alpha_{1} d \alpha_{2} d \alpha_{3} \frac{1}{a^{2}} e^{\frac{-i}{a}\left(\frac{z^{2}}{4}-z \cdot\left(\alpha_{2} p^{\prime}+\alpha_{3} p-\frac{\tilde{q}}{2}\right)\right)} e^{\frac{i}{a}\left(\alpha_{2}+\alpha_{3}\right) p \cdot \tilde{q}} \\
& \times \exp \left[-i\left(m^{2}\left(\alpha_{1}-\alpha_{2}-\alpha_{3}\right)+m_{\gamma}^{2}\left(\alpha_{2}+\alpha_{3}\right)+\frac{1}{a}\left(m^{2}\left(\alpha_{2}+\alpha_{3}\right)^{2}-\alpha_{2} \alpha_{3} q^{2}\right)-\frac{1}{a \Lambda_{e f f}^{2}}\right)\right],
\end{aligned}
$$


Producing the numerator through the derivatives over the auxiliary field, a Wick rotation, rescaling the $\alpha_{i}$ 's and finally inserting the identity $1=\int_{0}^{\infty} \frac{d \rho}{\rho} \delta\left(1-\sum \alpha_{i}\right)$, the expression $\Gamma_{\mu}^{(b 2)}$ is found to be

$$
\begin{aligned}
\Gamma_{\mu}^{(b 2)} & =\frac{-\alpha e^{\frac{i}{2} p \times p^{\prime}}}{\pi} \int_{0}^{1} d \alpha_{1} d \alpha_{2} d \alpha_{3} \delta\left(1-\sum \alpha_{i}\right) e^{i\left(\alpha_{2}+\alpha_{3}\right) p \cdot \tilde{q}} e^{-i p \times p^{\prime}} \times \int d \rho \\
& \exp \left[-\rho\left(m^{2}\left(\alpha_{1}-\alpha_{2}-\alpha_{3}\right)+m_{\gamma}^{2}\left(\alpha_{2}+\alpha_{3}\right)+m^{2}\left(\alpha_{2}+\alpha_{3}\right)^{2}-\alpha_{2} \alpha_{3} q^{2}\right)-\frac{1}{\rho \Lambda_{\text {eff }}^{2}}\right] \\
& \times\left(\tilde{A}_{\mu}+\frac{\tilde{B}_{\mu}}{\rho}+\frac{\tilde{C}_{\mu}}{\rho^{2}}\right),
\end{aligned}
$$

where now, $\tilde{A}_{\mu}, \tilde{B}_{\mu}$ and $\tilde{C}_{\mu}$ are,

$$
\begin{gathered}
\tilde{A}^{\mu}=\frac{1}{2} \gamma_{\mu}\left[\left(\alpha_{2}+\alpha_{3}\right)\left(p^{\prime}+p\right)^{2}-3 m^{2}-m^{2}\left(\alpha_{2}+\alpha_{3}\right)^{2}+\alpha_{2} \alpha_{3} q^{2}\right]+ \\
+\frac{m \alpha_{1}}{2}\left(\alpha_{2}+\alpha_{3}\right)\left(p^{\prime}+p\right)_{\mu}, \\
i \tilde{B}_{\mu}=\frac{3 i}{2} \gamma_{\mu}+\frac{1}{2}\left(m \tilde{q}_{\mu}+\gamma_{\mu} p \cdot \tilde{q}\right)\left(2-\alpha_{2}-\alpha_{3}\right)-\frac{\gamma \cdot \tilde{q}}{4}\left(p^{\prime}+p\right)_{\mu}\left(1+\alpha_{2}+\alpha_{3}\right), \\
\tilde{C}_{\mu}=\frac{\gamma \cdot \tilde{q} \tilde{q_{\mu}}}{4}+\frac{\gamma_{\mu} \tilde{q} \cdot \tilde{q}}{8} .
\end{gathered}
$$

Integration over $\rho$ leads to

$$
\begin{aligned}
\Gamma_{\mu}^{(b 2)=} & \frac{-\alpha e^{\frac{i}{2} p \times p^{\prime}}}{\pi} \int_{0}^{1} d \alpha_{1} d \alpha_{2} d \alpha_{3} \delta\left(1-\sum \alpha_{i}\right) e^{i\left(\alpha_{2}+\alpha_{3}\right) p \cdot \tilde{q}} e^{i p^{\prime} \cdot p} \times \\
& \times\left(\frac{2 \tilde{A}_{\mu} K_{1}(2 \sqrt{Y})}{\sqrt{Y} \Lambda_{e f f}^{2}}+2 \tilde{B}_{\mu} K_{0}(\sqrt{Y})+\tilde{C}_{\mu} \sqrt{Y} \Lambda_{e f f}^{2} K_{1}(2 \sqrt{Y})\right),
\end{aligned}
$$

with

$$
Y \equiv \frac{m^{2}\left(\alpha_{1}-\alpha_{2}-\alpha_{3}\right)+m_{\gamma}^{2}\left(\alpha_{2}+\alpha_{3}\right)+m^{2}\left(\alpha_{2}+\alpha_{3}\right)^{2}-\alpha_{2} \alpha_{3} q^{2}}{\Lambda_{\text {eff }}^{2}}
$$

The $\Gamma_{\mu}^{(b 1)}$ term, can be easily recovered from the above expression by setting any term proportional to $\theta^{\mu \nu}$ (i.e. $\tilde{q}^{\mu}$ ) to zero. Then we have,

$$
\Gamma_{\mu}^{(b 1)}=\frac{-\alpha e^{\frac{i}{2} p \times p^{\prime}}}{\pi} \int_{0}^{1} d \alpha_{1} d \alpha_{2} d \alpha_{3} \delta\left(1-\sum \alpha_{i}\right)\left(\frac{2 \bar{A}_{\mu} K_{1}(2 \sqrt{Z})}{\sqrt{Z} \Lambda^{2}}+2 \bar{B}_{\mu} K_{0}(2 \sqrt{Z})\right)
$$


while $Z$ is now,

$$
Z \equiv \frac{m^{2}\left(\alpha_{1}-\alpha_{2}-\alpha_{3}\right)+m_{\gamma}^{2}\left(\alpha_{2}+\alpha_{3}\right)+m^{2}\left(\alpha_{2}+\alpha_{3}\right)^{2}-\alpha_{2} \alpha_{3} q^{2}}{\Lambda^{2}} .
$$

and,

$$
\begin{aligned}
\bar{A}^{\mu}= & \frac{1}{2} \gamma_{\mu}\left(\left(\alpha_{2}+\alpha_{3}\right)\left(p^{\prime}+p\right)^{2}-3 m^{2}-m^{2}\left(\alpha_{2}+\alpha_{3}\right)^{2}+\alpha_{2} \alpha_{3} q^{2}\right)+ \\
& \frac{m \alpha_{1}}{2}\left(\alpha_{2}+\alpha_{3}\right)\left(p^{\prime}+p\right)_{\mu} \\
\bar{B}_{\mu}= & \frac{3}{2} \gamma_{\mu} .
\end{aligned}
$$

\subsection{Renormalization}

Now we try to look at the divergences appearing in our diagrams. In the case of usual QED we have a logarithmic UV divergence, and the problem of IR divergence was fixed by introducing the photon a finite mass, $m_{\gamma}$. Looking at our expressions of the form factors , we see that they contain $K_{0}$, and $K_{1}$, and both of the functions contain either $\frac{1}{\Lambda_{e f f}^{2}}$ or $\frac{1}{\Lambda^{2}}$ in their arguments. Taking the high energy limit $\Lambda^{2} \rightarrow \infty$, or the low energy limit $q \rightarrow 0$ simultaneously we see that all terms containing $K_{1}$ are finite, but there appear to be a logarithmic divergence due to $K_{0}$. Therefore, we recover the same logarithmic divergence of usual QED when taking the IR limit. The noncommutative QED was shown to be renormalizable up to the one loop level by adding the relevant counter terms [8], so we can safely drop the singular parts in $K_{0}$, and keeping only the finite parts. Now taking the $\Lambda \rightarrow \infty$ limit, and dropping the divergent parts the renormalized Gamma functions can be written as,

$$
\begin{gathered}
\Gamma_{\mu(U V-r e n)}^{(a)}=\frac{-\alpha e^{\frac{i}{2} p \times p^{\prime}}}{\pi} \int_{0}^{1} d \alpha_{1} d \alpha_{2} d \alpha_{3} \delta\left(1-\sum \alpha_{i}\right) e^{-i\left(\alpha_{2}+\alpha_{3}\right) p \cdot \tilde{q}} \times \\
\times\left(\frac{A_{\mu}}{\alpha_{1} m_{\gamma}^{2}+\left(\alpha_{2}+\alpha_{3}\right)^{2} m^{2}-\alpha_{2} \alpha_{3} q^{2}}-2 B_{\mu} \gamma_{E u l e r}+\Lambda_{e f f}^{2} C_{\mu}\right), \\
\Gamma_{\mu(U V-r e n)}^{(b 1)}=\frac{-\alpha e^{\frac{i}{2} p \times p^{\prime}}}{\pi} \int_{0}^{1} d \alpha_{1} d \alpha_{2} d \alpha_{3} \delta\left(1-\sum \alpha_{i}\right) \times \\
\times\left(\frac{\bar{A}_{\mu}}{m^{2}\left(\alpha_{1}-\alpha_{2}-\alpha_{3}\right)+m_{\gamma}^{2}\left(\alpha_{2}+\alpha_{3}\right)+m^{2}\left(\alpha_{2}+\alpha_{3}\right)^{2}-\alpha_{2} \alpha_{3} q^{2}}-2 \bar{B}_{\mu} \gamma_{\text {Euller }}\right),
\end{gathered}
$$


and

$$
\begin{gathered}
\Gamma_{\mu(U V-r e n)}^{(b 2)}=\frac{-\alpha e^{\frac{i}{2} p \times p^{\prime}}}{\pi} \int_{0}^{1} d \alpha_{1} d \alpha_{2} d \alpha_{3} \delta\left(1-\sum \alpha_{i}\right) e^{i\left(\alpha_{2}+\alpha_{3}\right) p \cdot \tilde{q}} e^{-i p \times p^{\prime}} \times \\
\left(\frac{\tilde{A}_{\mu}}{m^{2}\left(\alpha_{1}-\alpha_{2}-\alpha_{3}\right)+m_{\gamma}^{2}\left(\alpha_{2}+\alpha_{3}\right)+m^{2}\left(\alpha_{2}+\alpha_{3}\right)^{2}-\alpha_{2} \alpha_{3} q^{2}}-2 \tilde{B}_{\mu} \gamma_{\text {Euller }}+\tilde{C}_{\mu} \Lambda_{e f f}^{2}\right) .
\end{gathered}
$$

Finally the full renormalized Gamma function for the electron-photon vertex at the one loop level is

$$
\begin{aligned}
\Gamma_{\mu(U V-r e n)}= & \frac{-\alpha e^{\frac{i}{2} p \times p^{\prime}}}{\pi} \int_{0}^{1} d \alpha_{1} d \alpha_{2} d \alpha_{3} \delta\left(1-\sum \alpha_{i}\right) \times \\
& \times\left(\frac{A_{\mu} e^{-i\left(\alpha_{2}+\alpha_{3}\right) p \cdot \tilde{q}}}{\alpha_{1} m_{\gamma}^{2}+\left(\alpha_{2}+\alpha_{3}\right)^{2} m^{2}-\alpha_{2} \alpha_{3} q^{2}}+\right. \\
& +\frac{\bar{A}_{\mu}\left(1-e^{i\left(\alpha_{2}+\alpha_{3}\right) p \cdot \tilde{q}} e^{-i p \times p^{\prime}}\right)}{m^{2}\left(\alpha_{1}-\alpha_{2}-\alpha_{3}\right)+m_{\gamma}^{2}\left(\alpha_{2}+\alpha_{3}\right)+m^{2}\left(\alpha_{2}+\alpha_{3}\right)^{2}-\alpha_{2} \alpha_{3} q^{2}}- \\
& -2 \gamma_{E u l e r}\left(B_{\mu} e^{-i\left(\alpha_{2}+\alpha_{3}\right) p \cdot \tilde{q}}+\bar{B}_{\mu}-\tilde{B}_{\mu} e^{i\left(\alpha_{2}+\alpha_{3}\right) p \cdot \tilde{q}} e^{-i p \times p^{\prime}}\right)+ \\
& \left.+\Lambda_{e f f}^{2}\left(C_{\mu} e^{-i\left(\alpha_{2}+\alpha_{3}\right) p \cdot \tilde{q}}-\tilde{C}_{\mu} e^{i\left(\alpha_{2}+\alpha_{3}\right) p \cdot \tilde{q}} e^{-i p \times p^{\prime}}\right)\right) .
\end{aligned}
$$

In equation (3.30), we have given the UV - renormalized gamma function, but we can see that in the last term we still keep the cut-off in the expression, and this can be understood by the UV/IR mixing. In order this we will discuss both limits applied to this term, first considering the UV limit, i.e. $\frac{1}{\Lambda^{2}} \ll \tilde{q} \cdot \tilde{q}\left(\Lambda_{\text {eff }}^{2} \sim \frac{1}{\tilde{q} \cdot \tilde{q}}\right)$, we see that the term is finite, but when taking the IR limit first, i.e. $\frac{1}{\Lambda^{2}} \gg \tilde{q} . \tilde{q}$ then $\Lambda_{\text {eff }}^{2} \sim \frac{1}{\Lambda^{2}}$, so it may seem that this term will lead to an IR divergence. However, $C_{\mu}$ terms contain two type of terms, both proportional to $\tilde{q}^{2}$, and since $\Lambda^{2} \tilde{q}^{2} \ll 1$, in the IR limit, this term will be totally irrelevant. One should note that order of taking the $\Lambda^{2} \rightarrow \infty$ and $q \rightarrow 0$ is very important in our arguments; this is a generic feature of NCFT's and is called IR/UV mixing [6, 7]. Hence 
the fully renormalized vertex function is,

$$
\begin{aligned}
\Gamma_{\mu(\text { ren })}= & \frac{-\alpha e^{\frac{i}{2} p \times p^{\prime}}}{\pi} \int_{0}^{1} d \alpha_{1} d \alpha_{2} d \alpha_{3} \delta\left(1-\sum \alpha_{i}\right) \times \\
& \times\left(\frac{A_{\mu} e^{-i\left(\alpha_{2}+\alpha_{3}\right) p \cdot \tilde{q}}}{\alpha_{1} m_{\gamma}^{2}+\left(\alpha_{2}+\alpha_{3}\right)^{2} m^{2}-\alpha_{2} \alpha_{3} q^{2}}+\right. \\
& +\frac{\bar{A}_{\mu}\left(1-e^{i\left(\alpha_{2}+\alpha_{3}\right) p \cdot \tilde{q}} e^{-i p \times p^{\prime}}\right)}{m^{2}\left(\alpha_{1}-\alpha_{2}-\alpha_{3}\right)+m_{\gamma}^{2}\left(\alpha_{2}+\alpha_{3}\right)+m^{2}\left(\alpha_{2}+\alpha_{3}\right)^{2}-\alpha_{2} \alpha_{3} q^{2}}- \\
& \left.-2 \gamma_{\text {Euler }}\left(B_{\mu} e^{-i\left(\alpha_{2}+\alpha_{3}\right) p \cdot \tilde{q}}+\bar{B}_{\mu}-\tilde{B}_{\mu} e^{i\left(\alpha_{2}+\alpha_{3}\right) p \cdot \tilde{q}} e^{-i p \times p^{\prime}}\right)\right)
\end{aligned}
$$

For the moment we can see that $\Gamma^{\mu}$ can be written in the form,

$$
\Gamma^{\mu}=E_{1} \gamma^{\mu}+H_{1}\left(p^{\prime}+p\right)^{\mu}+G_{1} \tilde{q}^{\mu}+E_{2} \gamma^{\mu} p \cdot \tilde{q}+H_{3}\left(p^{\prime}+p\right)^{\mu} \gamma \cdot \tilde{q} .
$$

Collecting the different cofficients of $\gamma^{\mu},\left(p^{\prime}+p\right)^{\mu}, \tilde{q}^{\mu}, \gamma^{\mu} p . \tilde{q}^{\mu},\left(p^{\prime}+p\right)^{\mu} \gamma \cdot \tilde{q}$, we have

$$
\begin{aligned}
& E_{1}=\frac{-\alpha e^{\frac{i}{2} p \times p^{\prime}}}{\pi} \int_{0}^{1} d \alpha_{1} d \alpha_{2} d \alpha_{3} \delta\left(1-\sum \alpha_{i}\right) \times\left(1-e^{i\left(\alpha_{2}+\alpha_{3}\right) p \cdot \tilde{q}} e^{-i p \times p^{\prime}}\right) \\
& \times\left\{\left[\frac{\left.\left(2 p^{\prime} \cdot p-\left(\alpha_{2}+\alpha_{3}\right)\left(p^{\prime}+p\right)^{2}\right)+m^{2}\left(\alpha_{2}+\alpha_{3}\right)^{2}-\alpha_{2} \alpha_{3} q^{2}\right)}{2\left(\alpha_{1} m_{\gamma}^{2}+\left(\alpha_{2}+\alpha_{3}\right)^{2} m^{2}-\alpha_{2} \alpha_{3} q^{2}\right)}+\gamma_{\text {Euller }}\right] e^{-i\left(\alpha_{2}+\alpha_{3}\right) p \cdot \tilde{q}}\right. \\
& \left.+\left[\frac{\left(\left(\alpha_{2}+\alpha_{3}\right)\left(p^{\prime}+p\right)^{2}-3 m^{2}-m^{2}\left(\alpha_{2}+\alpha_{3}\right)^{2}+\alpha_{2} \alpha_{3} q^{2}\right)}{m^{2}\left(\alpha_{1}-\alpha_{2}-\alpha_{3}\right)+m_{\gamma}^{2}\left(\alpha_{2}+\alpha_{3}\right)+m^{2}\left(\alpha_{2}+\alpha_{3}\right)^{2}-\alpha_{2} \alpha_{3} q^{2}}-\frac{3 \gamma_{\text {Euller }}}{2}\right]\right\}, \\
& H_{1}=\frac{-\alpha e^{\frac{i}{2} p \times p^{\prime}}}{\pi} \int_{0}^{1} d \alpha_{1} d \alpha_{2} d \alpha_{3} \delta\left(1-\sum \alpha_{i}\right) \times \\
& \times\left\{\frac{m \alpha_{1}\left(\alpha_{2}+\alpha_{3}\right) e^{i\left(\alpha_{2}+\alpha_{3}\right) p . \tilde{q}}}{\alpha_{1} m_{\gamma}^{2}+\left(\alpha_{2}+\alpha_{3}\right)^{2} m^{2}-\alpha_{2} \alpha_{3} q^{2}}+\right. \\
& \left.+\frac{m \alpha_{1}\left(\alpha_{2}+\alpha_{3}\right)\left(1-e^{i\left(\alpha_{2}+\alpha_{3}\right) p \cdot \tilde{q}} e^{-i p \times p^{\prime}}\right)}{m^{2}\left(\alpha_{1}-\alpha_{2}-\alpha_{3}\right)+m_{\gamma}^{2}\left(\alpha_{2}+\alpha_{3}\right)+m^{2}\left(\alpha_{2}+\alpha_{3}\right)^{2}-\alpha_{2} \alpha_{3} q^{2}}\right\}, \\
& G_{1}=\frac{-\alpha e^{\frac{i}{2} p \times p^{\prime}}}{\pi} i m \gamma_{E u l l e r} \int_{0}^{1} d \alpha_{1} d \alpha_{2} d \alpha_{3} \delta\left(1-\sum \alpha_{i}\right) \times \\
& \times\left(\left(1+\alpha_{2}+\alpha_{3}\right) e^{-i\left(\alpha_{2}+\alpha_{3}\right) p \cdot \tilde{q}}-\left(2-\alpha_{2}-\alpha_{3}\right) e^{i\left(\alpha_{2}+\alpha_{3}\right) p \cdot \tilde{q}} e^{-i p \times p^{\prime}}\right), \\
& E_{2}=\frac{-\alpha e^{\frac{i}{2} p \times p^{\prime}}}{\pi}\left(i \gamma_{E u l l e r}\right) \int_{0}^{1} d \alpha_{1} d \alpha_{2} d \alpha_{3} \delta\left(1-\sum \alpha_{i}\right)\left(1-e^{-i p \times p^{\prime}}\right)\left(2-\alpha_{2}-\alpha_{3}\right) e^{-i\left(\alpha_{2}+\alpha_{3}\right) p \cdot \tilde{q}} \\
& H_{3}=\frac{-\alpha e^{\frac{i}{2} p \times p^{\prime}}}{\pi} \frac{i \gamma_{\text {Euller }}}{2} \int_{0}^{1} d \alpha_{1} d \alpha_{2} d \alpha_{3} \delta\left(1-\sum \alpha_{i}\right) \times \\
& \left(\left(2-\alpha_{2}-\alpha_{3}\right) e^{-i\left(\alpha_{2}+\alpha_{3}\right) p \cdot \tilde{q}}+\left(1+\alpha_{2}+\alpha_{3}\right) e^{i\left(\alpha_{2}+\alpha_{3}\right) p \cdot \tilde{q}} e^{-i p \times p^{\prime}}\right) \text {. }
\end{aligned}
$$


In the next section we will study the physical significance of these factors.

The only comment we would like to make in this part is the appearance of extra factors of $e^{-i p \times p^{\prime}}$ in the above expressions. More precisely, our vertex function in the one loop level contains two different terms, one proportional to $e^{\frac{i}{2} p \times p^{\prime}}$ and the other proportional to $e^{-\frac{i}{2} p \times p^{\prime}}$. This can be understood if we note that our thoery is not CP invariant and if we change the arrow of time in our diagrams we will find the same expression, but we also should change $\theta$ to $-\theta$ 14.

\section{The electron-photon vertex function}

In this section we give a formal discussion of the electron-photon vertex structure found in NCQED. So, first we review the similar argument in the usual QED. The physical meaning of the different terms appearing in our vertex from the calculation in the previous section will be discussed later.

\subsection{Electron-photon vertex in usual QED}

In this section we briefly present the structure of the electron-photon vertex in the usual QED. Considering the radiative corrections to the vertex, it can be written in the following a diagrammatic form

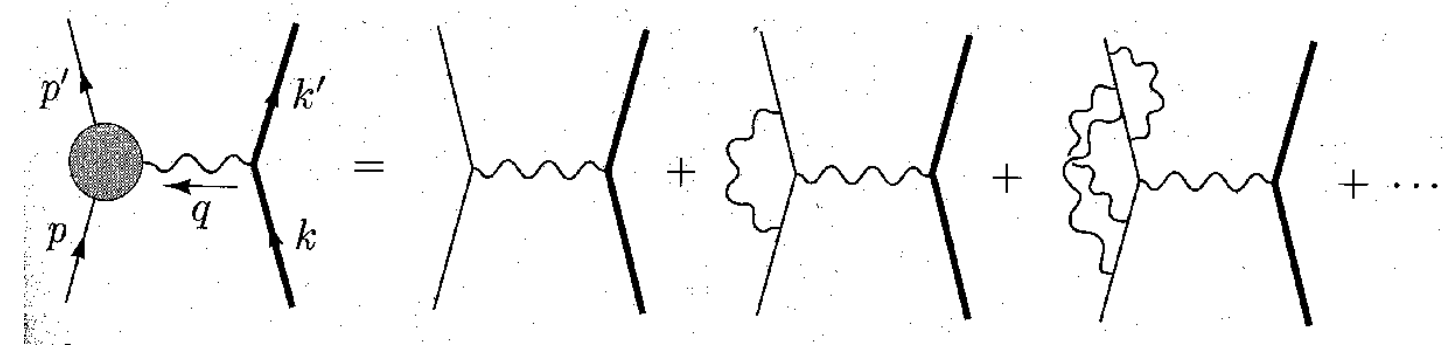

where the shaded circle can be expressed as

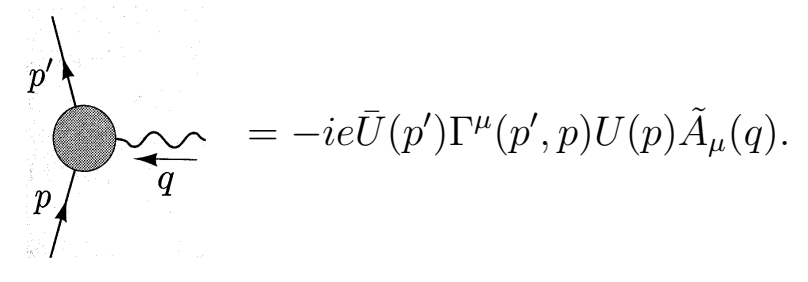

We observe that in the leading order of perturbation $\Gamma^{\mu} \rightarrow \gamma^{\mu}$. 
The list of available vectors and scalars is only consisting of $\left(p_{\mu}, p_{\mu}^{\prime}, \gamma_{\mu}, q^{2}, m, e\right)$. So, the most general form of the vertex function can be written as p

$$
\Gamma^{\mu}=A \gamma^{\mu}+B\left(p^{\prime}+p\right)^{\mu}+C\left(p^{\prime}-p\right)^{\mu},
$$

where A, B, C can be any scalars formed out of previous list. Appealing to Ward identity i.e. $q^{\mu} \Gamma_{\mu}=0$, we get

$$
0=A \not q+B\left(p^{\prime}+p\right) \cdot q+C\left(p^{\prime}-p\right) \cdot q .
$$

Since, $\bar{U}\left(p^{\prime}\right) \not q U(p)=0,\left(p^{\prime}+p\right) \cdot q=0$ and $\quad\left(p^{\prime}-p\right) \cdot q \neq 0$, we conclude that to satisfy the Ward identity, $C=0$. Therefore, $\Gamma^{\mu}$ for QED takes the following form,

$$
\Gamma^{\mu}=A \gamma^{\mu}+B\left(p^{\prime}+p\right)^{\mu}
$$

using the Gordon identity we can write the above expression as,

$$
\Gamma^{\mu}=F_{1}\left(q^{2}\right) \gamma^{\mu}+\frac{i \sigma^{\mu \nu} q_{\nu}}{2 m} F_{2}\left(q^{2}\right)
$$

$F_{1}\left(q^{2}\right)$ and $F_{2}\left(q^{2}\right)$ which usually are known as form factors, are functions of $\mathrm{m}$, e and $q^{2}$ depending on the diagram. One can see that at the tree level we have $\Gamma^{\mu} \rightarrow \gamma^{\mu}$, hence $F_{1}\left(q^{2}\right)=1$ and $F_{2}\left(q^{2}\right)=0$.

To give an interpretation to the above form factors we will couple this vertex to external slowly varying electric and magnetic fields at the tree level.

\section{The electron electric charge}

Let us consider the Coulomb scattering cross section, from a slowly varying electric field, i.e. we can take the limit $q^{2}=0$. The corresponding scattering amplitude can be written as

$$
-i M=-i e F_{1}(0) \tilde{\Phi}(q) \xi^{\dagger} \xi
$$

where we have used the non-relativistic approximation for the spinor field,

$$
U(p)=\frac{1}{\sqrt{2}}\left(\begin{array}{l}
\left(\begin{array}{l}
1-\frac{\vec{p} \cdot \vec{\sigma}}{2 m} \\
1+\frac{\vec{p} \cdot \vec{\sigma}}{2 m}
\end{array}\right) \xi
\end{array}\right) .
$$

\footnotetext{
${ }^{5}$ We have not included $\gamma^{5}$ because of the parity conservation.
} 
In the Born approximation $V(x)=e F_{1}(0) \Phi(x)$, hence we can identify $F_{1}(0)$ as the electric charge in units of e, and since $F_{1}(0)=1$ at the tree level, radiative corrections to $F_{1}\left(q^{2}\right)$ should vanish at $q^{2}=0$.

\section{The electron magnetic moment}

We now repeat the analysis for an electron scattering from a static vector potential. The amplitude of scattering from this field is

$$
i M=i e\left[\bar{U}\left(p^{\prime}\right)\left(\gamma^{i} F_{1}+\frac{i \sigma^{i \nu}}{2 m} q_{\nu} F_{2}\right) U(p)\right] \tilde{A}^{i}(q)
$$

Inserting the non-relativistic expansion for the spinors (4.7), and keeping terms first order in momenta we obtain,

$$
i M=-i e \xi^{\dagger}\left(\frac{-\sigma_{k}}{2 m}\left[F_{1}(0)+F_{2}(0)\right]\right) \xi \tilde{B}^{k}(q),
$$

where

$$
\tilde{B}^{k}(q)=-i \epsilon^{i j k} q_{i} \tilde{A}_{j}(q)
$$

is the Fourier transform of the magnetic field produced by $A(x)$.

Again we interpret $M$ as the Born approximation to the scattering of the electron from a potential. The potential is just that of a magnetic moment interaction, $V(x)=$ $-\langle\mu\rangle \cdot B(x)$, where,

$$
\langle\vec{\mu}\rangle=\frac{e}{m}\left[F_{1}(0)+F_{2}(0)\right] \xi^{\dagger} \frac{\vec{\sigma}}{2} \xi
$$

This expression for the magnetic moment of the electron can be rewritten in the standard form

$$
\vec{\mu}=g\left(\frac{e}{2 m}\right) \vec{S}
$$

where $\vec{S}$ is the electron spin. The coefficient $g$ is

$$
g=2\left[F_{1}(0)+F_{2}(0)\right]=2+2 F_{2}(0) .
$$

Along our previous argument for the leading order of $F_{1}$ and $F_{2}$, we see that to the leading order (classical level) the magnetic moment of a Dirac particle is 2 . 


\subsection{Dipole moment at the tree level in NCQED}

The calculation of the vertex function at the tree level goes exactly as in the case of usual QED, except for the extra phase $e^{\frac{i}{2} p \times p^{\prime}}$ appearing due to the star product, i.e.

$$
\begin{aligned}
\Gamma^{\mu} & =e^{\frac{i}{2} p \times p^{\prime}} \gamma^{\mu} \\
& =e^{\frac{i}{2} p \cdot \tilde{q}} \gamma^{\mu}
\end{aligned}
$$

Using a power expansion of the exponential and keeping only the first two terms, we see that the first term in the expansion which is $\theta$ independent gives rise to the usual result in QED, i.e. we get the same electric charge and magnetic dipole moment. The second term which is proportional to $\theta$ will give rise to an electric dipole moment and couples to the external electric field, $E$, as $\langle P\rangle . E$, where

$$
\left\langle P_{i}\right\rangle=\frac{1}{2} e \tilde{p}_{i}=\frac{1}{2} e \theta_{j i} p_{j}
$$

This term will also contribute to higher-pole moments when coupled to an external electromagnetic field.

\subsection{The electron-photon vertex structure in NCQED}

In the above we discussed the structure of the electron-photon vertex, in the case of usual QED. The list of vectors and scaler appearing in the vertex function was restricted to $\left(\gamma^{\mu},\left(p^{\prime}+p\right)^{\mu}, q^{2}, m, e\right)$. In the case of NCQED, and due to the presence of $\theta^{\mu \nu}$, our previous list of independent vectors and scalars will be extended to include three other scalars

$$
\left(q^{2}, m, e, \tilde{q} \cdot \tilde{q}, \gamma \cdot \tilde{q}, p \cdot \tilde{q}\right)
$$

and the list for the vectors will be

$$
\left(\gamma^{\mu},\left(p^{\prime}+p\right)^{\mu}, q^{\mu}, \tilde{q}^{\mu}\right)
$$

The most general structure of the vertex function which is compatible with the Ward identity, is

$$
\Gamma^{\mu}=E \gamma^{\mu}+H\left(p^{\prime}+p\right)^{\mu}+G \tilde{q}^{\mu}
$$

where E, F and G are scalars formed from the list of our previous scalars, except for $\tilde{q} . \tilde{q}$, which as discussed in the previous section will not appear in the first loop. Up to the 
one loop approximation the coefficients $\mathrm{E}, \mathrm{F}$ and $\mathrm{G}$ may be expanded to be written as functions of $q^{2}, p . \tilde{q}$ and $\gamma \cdot \tilde{q}$. The form factors $F_{1}\left(q^{2}\right)$ and $F_{2}\left(q^{2}\right)$ can be picked directly from $E_{1}$ and $H_{1}$ using the Gordon identity. From the results of the previous calculations one can see that the only non-zero coefficients are $E_{1}, E_{2}, H_{1}, H_{3}$ and $G_{1}$, i.e.

$$
\Gamma^{\mu}=E_{1} \gamma^{\mu}+H_{1}\left(p^{\prime}+p\right)^{\mu}+G_{1} \tilde{q}^{\mu}+E_{2} \gamma^{\mu} p \cdot \tilde{q}+H_{3}\left(p^{\prime}+p\right)^{\mu} \gamma \cdot \tilde{q}
$$

So what is left now is to give a physical interpretation to those coefficients proportional to $\theta$. We will use the non-relativistic limit, to compute $\bar{U}\left(p^{\prime}\right) \Gamma^{\mu} U(p)$. Keeping terms up to second order of momentum we find,

- $G_{1}$, coefficients of $\tilde{q}_{\mu}$ :

This term will give a contribution to the magnetic moment. The corresponding effective interaction potential with the external magnetic filed is $V(x)=-\langle\mu\rangle \cdot B(x)$, where

$$
\langle\vec{\mu}\rangle=\frac{G_{1}}{i} \vec{\theta}, \quad \theta_{i} \equiv \epsilon_{i j k} \theta_{j k}
$$

As we see this magnetic moment does not depend on spin.

- $E_{2}$, coefficients of $\gamma^{\mu} p . \tilde{q}$ :

This term will vanish in the non-relativistic limit, but it will give contribution to higher moments when higher orders of momentum are considered.

- $H_{3}$, coefficients of $\left(p^{\prime}+p\right)^{\mu} \gamma \cdot \tilde{q}$ :

This term will give rise to an electric dipole moment of the form

$$
\langle P\rangle_{i}=2 i H_{3} \tilde{p}_{i}
$$

Since we are going to work in the low momentum limit, we can use the series expansion of $e^{-i\left(\alpha_{2}+\alpha_{3}\right) p \cdot \tilde{q}}, e^{i\left(\alpha_{2}+\alpha_{3}\right) p \cdot \tilde{q}}$ and $e^{i p^{\prime} \times p}$ and keep only the first term of the expansion. In 
this limit

$$
\begin{aligned}
E_{1}= & \frac{-\alpha e^{\frac{i}{2} p \times p^{\prime}}}{\pi} \int_{0}^{1} d \alpha_{1} d \alpha_{2} d \alpha_{3} \delta\left(1-\sum \alpha_{i}\right) \times \\
& \times\left[\frac{\left.\left(2 p^{\prime} \cdot p-\left(\alpha_{2}+\alpha_{3}\right)\left(p^{\prime}+p\right)^{2}\right)+m^{2}\left(\alpha_{2}+\alpha_{3}\right)^{2}-\alpha_{2} \alpha_{3} q^{2}\right)}{2\left(\alpha_{1} m_{\gamma}^{2}+\left(\alpha_{2}+\alpha_{3}\right)^{2} m^{2}-\alpha_{2} \alpha_{3} q^{2}\right)}+\gamma_{\text {Euller }}\right], \\
H_{1}= & \frac{-\alpha e^{\frac{i}{2} p \times p^{\prime}}}{\pi} \int_{0}^{1} d \alpha_{1} d \alpha_{2} d \alpha_{3} \delta\left(1-\sum \alpha_{i}\right) \frac{m \alpha_{1}\left(\alpha_{2}+\alpha_{3}\right)}{\alpha_{1} m_{\gamma}^{2}+\left(\alpha_{2}+\alpha_{3}\right)^{2} m^{2}-\alpha_{2} \alpha_{3} q^{2}}, \\
G_{1}= & \frac{-\alpha e^{\frac{i}{2} p \times p^{\prime}}}{\pi} i m \gamma_{\text {Euller }} \int_{0}^{1} d \alpha_{1} d \alpha_{2} d \alpha_{3} \delta\left(1-\sum \alpha_{i}\right)\left(\alpha_{2}+\alpha_{3}-1\right), \\
H_{3}= & \frac{-\alpha e^{\frac{i}{2} p \times p^{\prime}}}{\pi} \frac{3 i \gamma_{\text {Euller }}}{2} \int_{0}^{1} d \alpha_{1} d \alpha_{2} d \alpha_{3} \delta\left(1-\sum \alpha_{i}\right) .
\end{aligned}
$$

Now we can apply the Gordon identity to $E_{1}$ and $H_{1}$, and identify the form factors $F_{1}\left(q^{2}\right)$ and $F_{2}\left(q^{2}\right)$ directly

$$
\begin{gathered}
F_{1}\left(q^{2}\right)=\frac{-\alpha e^{\frac{i}{2} p \times p^{\prime}}}{\pi} \int_{0}^{1} d \alpha_{1} d \alpha_{2} d \alpha_{3} \delta\left(1-\sum \alpha_{i}\right) \times \\
\times\left[\frac{2 m^{2}\left(1-\alpha_{2}-\alpha_{3}\right)-q^{2}\left(1-\alpha_{2}-\alpha_{3}\right)-m^{2}\left(\alpha_{2}+\alpha_{3}\right)^{2}-\alpha_{2} \alpha_{3} q^{2}}{2\left(\alpha_{1} m_{\gamma}^{2}+\left(\alpha_{2}+\alpha_{3}\right)^{2} m^{2}-\alpha_{2} \alpha_{3} q^{2}\right)}+\gamma_{\text {Euller }}\right],
\end{gathered}
$$

and

$$
F_{2}\left(q^{2}\right)=\frac{\alpha e^{\frac{i}{2} p \times p^{\prime}}}{\pi} \int_{0}^{1} d \alpha_{1} d \alpha_{2} d \alpha_{3} \delta\left(1-\sum \alpha_{i}\right) \frac{m^{2} \alpha_{1}\left(\alpha_{2}+\alpha_{3}\right)}{\alpha_{1} m_{\gamma}^{2}+\left(\alpha_{2}+\alpha_{3}\right)^{2} m^{2}-\alpha_{2} \alpha_{3} q^{2}} .
$$

As it is seen, apart from the pre-factor, $e^{\frac{i}{2} p \times p^{\prime}}$, the (4.20), (4.21) expressions are exactly the same as the usual QED.

The integration over the Schwinger parameters in $G_{1}$, and $H_{3}$ terms can be easily performed,

$$
\begin{aligned}
& G_{1}=\frac{\alpha e^{\frac{i}{2} p \times p^{\prime}}}{\pi}\left(\frac{i m \gamma_{\text {Euller }}}{6}\right) \\
& H_{3}=\frac{-\alpha e^{\frac{i}{2} p \times p^{\prime}}}{\pi}\left(\frac{3 i \gamma_{\text {Euller }}}{4}\right) .
\end{aligned}
$$

Altogether, we can write the interaction of a noncommutative electron and an external electromagnetic field in the form,

$$
V(x)=e \Phi+\langle\mu\rangle \cdot B+\langle P\rangle \cdot E
$$


where the first term is just a Coulomb potential, the second is the magnetic dipole moment and the last term is the electric dipole moment. The coefficients $\langle\mu\rangle$ and $\langle P\rangle$, in the low momentum approximation, and up to first loop are

$$
\begin{aligned}
\langle\vec{\mu}\rangle & =\frac{e}{m}\left[\left(F_{1}(0)+F_{2}(0)\right) \vec{S}+\frac{\alpha \gamma_{\text {Euller }}}{6 \pi} m^{2} \vec{\theta}\right] \\
\langle\vec{P}\rangle & =\frac{1}{4} e(\vec{\theta} \times \vec{p})\left(1+3 \alpha \gamma_{\text {Euller }}\right)
\end{aligned}
$$

\section{Concluding remarks}

In this work we have discussed some aspects of NCQED. First we introduced the theory by giving its action, and the corresponding basic Feynman graphs. We argued that the photon itself, similar to the moving noncommutative electron, even at classical level, shows some electric dipole effect. However, the dipole moment of the fermions compared to that of photon, is less by a factor of one half. This is due to the fact that in the NCYM action, the noncommutative effects appear as the Moyal-bracket, while in the fermionic sector it is just a star product.

Calculating the one loop contributions to the electron-photon vertex, we studied the electro-magnetic dipole moments of the electron. We showed that magnetic dipole moment of electron has now two parts, one spin dependent which will not receive any further corrections due to the noncommutativity, and the other spin independent, being proportional to $\theta$. In addition we also found the one loop contributions to the electric dipole moment.

Here we only studied the loop effects coming form the interaction vertex correction, however there are many other interesting phenomena coming from the vacuum polarization and corrections to propagators such as, Lamb shift, or the pair production threshold, which we postpone them to the future studies.

\section{Acknowledgements}

One of us, I.F. R. , would like to thank all the Professors of ICTP HEP Diploma Course, Ms. Concetta Mosca for her endless help throughout the year, UNESCO, the IAEA and Professor M.A. Virasoro, Director of ICTP, for their kind hospitality at the Centre during the Diploma course programme. M.M. Sh-J. would like to thank T. Krajewski for helpful comments. 
This research was partly supported by the EC contract no. ERBFMRX-CT 96-0090.

\section{A Some useful identities in *-product calculus}

Let $f, g$ be two arbitrary functions on non-commutative $R^{d}$ :

$$
f(x)=\int f(k) e^{i k \cdot x} d^{d} k, \quad g(x)=\int g(k) e^{i k \cdot x} d^{d} k .
$$

Then

$$
(f * g)(x)=\int f(k) g(l) e^{-i k \theta l / 2} e^{i(k+l) \cdot x} d^{d} k d^{d} l,
$$

where $k \theta l=k^{\mu} \theta_{\mu \nu} l^{\nu}$. From the above relation it is straightforward to see:

1) $g * f=\left.f * g\right|_{\theta \rightarrow-\theta}$, and hence $\{f, g\}_{M . B}=\left.f * g\right|_{\theta}-\left.f * g\right|_{-\theta}$.

2) $\int(f * g)(x) d^{d} x=\int(g * f)(x) d^{d} x=\int f g(x) d^{d} x$.

3) If we denote complex conjugation by c.c., then

$$
(f * g)^{\text {c.c. }}=g^{\text {c.c. }} * f^{\text {c.c. }} \text {. }
$$

If $h$ is another arbitrary function:

4) $(f * g) * h=f *(g * h) \equiv f * g * h$.

5) $\int(f * g * h)(x) d^{d} x=\int(h * f * g)(x) d^{d} x=\int(g * h * f)(x) d^{d} x$.

6) $\left.(f * g * h)\right|_{\theta}=\left.(h * g * f)\right|_{-\theta}$.

In other words the integration on the space coordinates, $x$, has the cyclic property, and it has all the properties of the $\operatorname{Tr}$ in the matrix calculus.

From 2) we learn that the kinetic part of the actions (which are quadratic in fields) is the same as their commutative version. So the free field propagators in commutative and nnoncommutative spaces are the same.

\section{References}

[1] H.S. Snyder, Quantized Space-Time, Phys. Rev. 71 (1947) 38; The Electromagnetic Field in Quantized Sapce-Time, Phys. Rev. 72 (1947) 68.

[2] A. Connes, M.R. Douglas, C. Hull, JHEP 9802 (1998) 008, hep-th/9711162.

[3] N. Seiberg, E. Witten, String Theory and Noncommutative Geometry, JHEP 9909 (1999) 032, hep-th/9908142, and references there in. 
[4] A. Gonzalez-Arroyo, C.P. Korthals Altes, Reduced Model for Large N Continuum Theories, Phys. Lett. B131 (1983) 396.

T. Filk, Divergences in a Field Theory on Quantum Space, Phys. Lett. B376 (1996) 53.

M. Chaichian, A. Demichev, P. Presnajder, Quantum Field Theory on Noncommutative Space-Times and the Persistence of Ultraviolet Divergences, Nucl. Phys. B 567 (2000) 360;

M.M. Sheikh-Jabbari, One Loop Renormalizability of Supersymmetric Yang-Mills Theories on Noncommutative Two-Torus, JHEP 9906 (1999) 015, hep-th/9903107;

C.P. Martin, D. Sanchez-Ruiz, The One-loop UV Divergent Structure of U(1) Yang-Mills Theory on Noncommutative $R^{4}$, Phys. Rev. Lett. 83 (1999) 476, hepth/9903077;

T. Krajewski, R. Wulkenhaar, Perturbative quantum gauge fields on the noncommutative torus, it Int. J. Mod. Phys. A15 (2000) 1011, hep-th/9903187.

H. Grosse, T. Krajewski, R. Wulkenhaar, Renormalization of Noncommutative YangMills Theories: A simple example, hep-th/0001182.

[5] I. Ya. Aref'eva, D.M. Belov, A.S. Koshelev, Two-Loop Diagrams in Noncommutaitve $\varphi_{4}^{4}$ Theory, Phys. Lett. B476 (2000) 431. hep-th/9912075.

[6] S. Minwalla, M. Van Raamsdonk, N. Seiberg, Noncommutative Perturbative Dynam$i c s$, hep-th/9912072;

M. Van Raamsdonk, N. Seiberg, Comments on Noncommutative Perturbative Dynamics, JHEP 0003 (2000) 035, hep-th/0002186.

[7] A. Matusis, L. Susskind, N. Toumbas, The IR/UV Connection in the Noncommutative Gauge Theories, hep-th/0002075.

[8] M. Hayakawa, Perturbative analysis on infrared and ultraviolet aspects of noncommutative $Q E D$ on $R^{4}$, hep-th/9912167.

[9] A. Armoni, Comments on Perturbative Dynamics of Non-Commutative Yang-Mills Theory, hep-th/0005208. 
[10] F. Ardalan, N. Sadooghi, Axial Anomaly in Non-Commutative QED on $R^{4}$, hepth/0002143;

J. M. Gracia-Bondia, C. P. Martin, Chiral Gauge Anomalies on Noncommutative $R^{4}$, Phys. Lett. B479 (2000) 321, hep-th/0002171;

L. Bonora, M. Schnabl, A. Tomasiello, A note on consistent anomalies in noncommutative YM theories, Phys. Lett. B485 (2000) 311, hep-th/0002210.

[11] L. Alvarez-Gaume, J.F. Barbon, Non-linear Vacuum Phenomena in Noncommutative $Q E D$, hep-th/0006209.

[12] L. Alvarez-Gaume, S. R. Wadia, Gauge Theory on a Quantum Phase Space, hepth/0006219.

[13] A. Micu, M.M. Sheikh-Jabbari, Noncommutative $\Phi^{4}$ Theory at Two Loops, hep-th/0008057.

[14] M.M. Sheikh-Jabbari, Discrete Symmetries (C, P, T) in Noncommutative Field Theories, Phys. Rev. Lett. 84 (2000) 5265, hep-th/000167

[15] I. Mocioiu, M. Pospelov, R. Roiban, Low-energy Limits on the Anti-symmetric Tensor Field Background on the Brane and on the Noncommutative Scale, hep$\mathrm{ph} / 0005191$.

[16] J. Gomis, T. Mehen, Space-Time Noncommutative Field Theories And Unitarity, hep-th/0005129.

M. Chaichian, A. Demichev, P. Presnajder, A. Tureanu, Space-Time Noncommutativity, Discreteness of Time and Unitarity, hep-th/0007156.

[17] N. Seiberg, L. Susskind, N. Toumbas, Space/Time Non-Commutativity and Causality, JHEP 0006 (2000) 044, hep-th/0005015.

O. Aharony, J. Gomis, T. Mehen, On Theories With Light-Like Noncommutativity, hep-th/0006236.

[18] M.M. Sheikh-Jabbari, Open Strings in a B-field Background as Electric Dipoles, Phys. Lett. B455 (1999) 129, hep-th/9901080. 
[19] I. S. Gradshteyn, I. M. Ryzhik, Tables of Integrals, Series and Products, Academic Press, New York, 1980.

[20] S. Weinberg, The Quantum Theory of Fields, Cambridge University press, 1996, vol. I \& II.

[21] C.Itzykson and J-B. Zuber, Quantum Field Theory, McGraw-Hill, 1985. 